May, 1993

\title{
Shape-invariant potentials and an associated coherent state
}

\author{
Takahiro Fukui \\ Yukawa Institute for Theoretical Physics \\ Kyoto University, Kyoto 606-01, Japan \\ N. Aizawa \\ Research Center for Nuclear Physics \\ Osaka University, Ibaraki, Osaka 567, Japan
}

\begin{abstract}
An algebraic treatment of shape-invariant potentials in supersymmetric quantum mechanics is discussed. By introducing an operator which reparametrizes wave functions, the shape-invariance condition can be related to a oscillator-like algebra. It makes possible to define a coherent state associated with the shapeinvariant potentials. For a large class of such potentials, it is shown that the introduced coherent state has the property of resolution of unity.
\end{abstract}


Coherent states are one of important concepts for physics today [1]. The original coherent state based on the Heisenberg-Weyl group is extended for a number of Lie groups with square integrable representations, and they have many applications in quantum mechanics. In particular, they are used as bases of coherent state path integrals $[2,3]$ or dynamical wavepackets for describing the quantum systems in semiclassical approximations [4]. Especially, if the hamiltonians have some dynamical symmetries, they can be described easily and intuitively by using the related coherent states as a basis. This is quite remarkable when we apply a semiclassical approximation to such systems; higher order quantum corrections can be included in the classical approximation [5]. There may exist several different dynamical symmetries if there exist several solvable potentials. Therefore, we often select different coherent states for different systems. However, it was shown [6] that a large class of these solvable potentials are characterised by a simple property, i.e., a discrete reparametrization invariance, called shape-invariance, introduced by means of the supersymmetric quantum mechanics [7]. It is a generalization of the old factorization method [8], in which possible six types of exactly solved potentials are classified. It is still an open question, however, to confirm the degree of its generalization and to classify the possible shape-invariant potentials.

Recently, Spiridonov analyzed a new class of shape-invariant potentials [9]. It is not under a change of parameters $a \rightarrow f(a)$ but under a dilatation of the dynamical varible $x \rightarrow q x$ that he demands, as a shape-invariance condition, the superpotentials are invariant. Therefore, such potentials have a property of self-similarity. Surprisingly, their dynamical symmetry is the quantum algebra $s u_{q}(1,1)$. The key idea is to introduce the dilatation operator $T_{q} f(x)=f(q x)$, which makes possible to express the condition of self-similarity as the commutation relation of the qoscillator, and it enables us to construct the $s u_{q}(1,1)$ generators. Then questions arise: Are there any algebra-like structure in the usual shape-invariant potentials as well as the self-similar ones? If so, is it possible to introduce anything like a coherent state describing a large class of such potentials simultaneously?

In this letter, a Lie algebra-like treatment of the shape-invariant potentials is developed. We first introduce an operator $T$ denoting reparametrization $\operatorname{Tg}(x, a)=$ $g\left(x, a_{1}\right)$, where $a_{1}=f(a)$, and then represent the shape-invariance condition as a commutation relation. Based on this, we define a coherent state associated with 
the shape-invariant potentials. It is shown that this coherent state has the property of resolution of unity for a large class of solvable potentials.

Let us introduce the superpotential $W(x, a)$ dependent on a parameter $a$ and assume that it satisfies the following shape-invariance condition [6]

$$
W^{2}(x, a)+W^{\prime}(x, a)=W^{2}\left(x, a_{1}\right)-W^{\prime}\left(x, a_{1}\right)+2 R\left(a_{1}\right),
$$

where $a_{1}=a-1 \equiv f(a)$ and $R$ is a function independent of $x$. Strictly speaking, shape-invariant potentials are not exhausted in this choice of parameters, namely, it is possible, for example, to introduce more than two parameters. This choice corresponds to the one discussed in Ref.[8]. However, we consider that factorization method in Ref.[8] is a prototype of the shape-invariance condition, and in this letter, we confine our attention to this case and illustrate our basic ideas.

The shape-invariance condition (1) can be rewritten in the following way. First, let $T$ be defined

$$
T|\phi(x, a)\rangle=\left|\phi\left(x, a_{1}\right)\right\rangle
$$

Namely, it is an operator which denotes the reparametrization of $a$. From this definition, operators $O(x, a)$, which depends on $a$, are transformed as

$$
O\left(x, a_{1}\right)\left|\phi\left(x, a_{1}\right)\right\rangle \equiv T O(x, a)|\phi(x, a)\rangle=T O(x, a) T^{-1}\left|\phi\left(x, a_{1}\right)\right\rangle
$$

where $T T^{-1}=1$, i.e., $T^{-1}|\phi(x, a)\rangle=\left|\phi\left(x, a_{-1}\right)\right\rangle$ with $a_{-1}=a+1$. Thus we have

$$
O\left(x, a_{1}\right)=T O(x, a) T^{-1}
$$

Next we define the hermitian conjugate of $T$. From the definition (2), we have the corresponding bra vector $\langle\phi(x, a)| T^{\dagger}=\left\langle\phi\left(x, a_{1}\right)\right|$. It should be noted here that the $T$ does not preserve the inner product in general; $\left\langle\phi\left(x, a_{1}\right) \mid \varphi\left(x, a_{1}\right)\right\rangle \neq$ $\langle\phi(x, a) \mid \varphi(x, a)\rangle$. We do not further enter into a mathematically rigorous definition of $T$, rather, we use it as a convenient tool for developing an algebraic treatment. 
By using $T$, the shape-invariance condition (1) is written as

$$
D(a) D^{\dagger}(a)=T\left\{D^{\dagger}(a) D(a)+R(a)\right\} T^{-1},
$$

where

$$
D(a) \equiv\left\{W(x, a)+\partial_{x}\right\} / \sqrt{2}
$$

Here and in the following, we do not denote explicitly $x$-dependence of functions and operators. With these, we define new operators [9]

$$
\begin{aligned}
& A_{+}(a)=D^{\dagger}(a) T, \\
& A_{-}(a)=T^{-1} D(a),
\end{aligned}
$$

Then the shape-invariance condition (4) is simply given by

$$
\left[A_{-}(a), A_{+}(a)\right]=R(a)
$$

Though this commutation relation resembles that of Heisenberg-Weyl algebra, it differs in the following points; i) $A_{-}$and $A_{+}$are not hermitian conjugate each other. ii) It is not a closed relation, namely, $A_{ \pm}$and $R$ are not commutative in general.

With these preparations, we examine the properties of the eigenstates of the hamiltonian $H_{0}=D^{\dagger}\left(a_{0}\right) D\left(a_{0}\right)$. We assume it has a zero energy state, i.e., there exist a normalizable state $\left|\psi_{0}\left(a_{0}\right)\right\rangle$ which satisfy

$$
D\left(a_{0}\right)\left|\psi_{0}\left(a_{0}\right)\right\rangle=A_{-}\left(a_{0}\right)\left|\psi_{0}\left(a_{0}\right)\right\rangle=0
$$

In the coordinate representation it is given by $\left\langle x \mid \psi_{0}\left(a_{0}\right)\right\rangle \propto \exp \left[-\int^{x} W\left(y, a_{0}\right) d y\right]$, which should be square integrable [7]. According to Gendenstein [6], we define the sequence of hamiltonians

$$
\begin{aligned}
& H_{0} \equiv A_{+}\left(a_{0}\right) A_{-}\left(a_{0}\right), \\
& H_{1} \equiv A_{-}\left(a_{1}\right) A_{+}\left(a_{1}\right)=A_{+}\left(a_{1}\right) A_{-}\left(a_{1}\right)+R\left(a_{1}\right), \\
& H_{2} \equiv A_{-}\left(a_{2}\right) A_{+}\left(a_{2}\right)+R\left(a_{1}\right)=A_{+}\left(a_{2}\right) A_{-}\left(a_{2}\right)+R\left(a_{1}\right)+R\left(a_{2}\right), \\
& \vdots \\
& H_{n} \equiv A_{-}\left(a_{n}\right) A_{+}\left(a_{n}\right)+\sum_{k=1}^{n-1} R\left(a_{k}\right)=A_{+}\left(a_{n}\right) A_{-}\left(a_{n}\right)+\sum_{k=1}^{n} R\left(a_{k}\right),
\end{aligned}
$$

where $a_{k} \equiv f\left(f\left(\cdots f\left(a_{0}\right) \cdots\right)\right)=a_{0}-k$. For these hamiltonians, we can see that 
$H_{n}$ and $H_{n+1}$ are superpartners, that is, they are isospectral except for the ground state of $H_{n}$.

Considering this property, we see that the eigenvalues and eigenstates of $H_{0}$ are given by

$$
\begin{aligned}
& E_{n}\left(a_{0}\right)=\sum_{k=1}^{n} R\left(a_{k}\right), \\
& \left|\psi_{n}\left(a_{0}\right)\right\rangle \propto\left\{A_{+}\left(a_{0}\right)\right\}^{n}\left|\psi_{0}\left(a_{0}\right)\right\rangle,
\end{aligned}
$$

provided there exist normalized states $\left|\psi\left(a_{k}\right)\right\rangle$ satisfying $A_{-}\left(a_{k}\right)\left|\psi\left(a_{k}\right)\right\rangle=0$ for $k=0, \ldots, n$. The last of Eq.(10) is easily verified by the commutation relation

$$
\left[H_{0},\left\{A_{+}\left(a_{0}\right)\right\}^{n}\right]=\left\{\sum_{k=1}^{n} R\left(a_{k}\right)\right\}\left\{A_{+}\left(a_{0}\right)\right\}^{n}
$$

which shows $H_{0}\left\{A_{+}\left(a_{0}\right)\right\}^{n}\left|\psi_{0}\left(a_{0}\right)\right\rangle=\left(\left\{A_{+}\left(a_{0}\right)\right\}^{n} H_{0}+\sum_{k=1}^{n} R\left(a_{k}\right)\left\{A_{+}\left(a_{0}\right)\right\}^{n}\right)$ $\left|\psi_{0}\left(a_{0}\right)\right\rangle=E_{n}\left(a_{0}\right)\left\{A_{+}\left(a_{0}\right)\right\}^{n}\left|\psi_{0}\left(a_{0}\right)\right\rangle$. It should be noted here that excited states are generated by one operator $A_{+}\left(a_{0}\right)$ and this makes possible to construct a coherent state. In terms of $D\left(a_{k}\right)$, the last equation can be rewritten as a more familar form

$$
\left|\psi_{n}\left(a_{0}\right)\right\rangle \propto D^{\dagger}\left(a_{0}\right) D^{\dagger}\left(a_{1}\right) \cdots D^{\dagger}\left(a_{n-1}\right)\left|\psi_{0}\left(a_{n}\right)\right\rangle
$$

The level scheme is summarized in Figure.

Next consider the normalization of the eigenstates. Let $\left|\psi_{n}\left(a_{k}\right)\right\rangle$ be the normalized eigenstates, then $\left|\psi_{n}\left(a_{k+1}\right)\right\rangle=T\left|\psi_{n}\left(a_{k}\right)\right\rangle$ with $\left\langle\psi_{n}\left(a_{k+1}\right) \mid \psi_{n}\left(a_{k+1}\right)\right\rangle=1$. Therefore

$$
A_{+}\left(a_{0}\right)\left|\psi_{n}\left(a_{0}\right)\right\rangle=D^{\dagger}\left(a_{0}\right)\left|\psi_{n}\left(a_{1}\right)\right\rangle=\sqrt{N_{n+1}\left(a_{0}\right)}\left|\psi_{n+1}\left(a_{0}\right)\right\rangle
$$

which shows

$$
\begin{aligned}
N_{n+1}\left(a_{0}\right) & =\left\langle\psi_{n}\left(a_{1}\right)\left|D\left(a_{0}\right) D^{\dagger}\left(a_{0}\right)\right| \psi_{n}\left(a_{1}\right)\right\rangle \\
& =\left\langle\psi_{n}\left(a_{1}\right)\left|H_{1}+R\left(a_{1}\right)\right| \psi_{n}\left(a_{1}\right)\right\rangle=\sum_{k=1}^{n+1} R\left(a_{k}\right)
\end{aligned}
$$


Thus we obtain

$$
\begin{aligned}
& A_{+}\left(a_{0}\right)\left|\psi_{n}\left(a_{0}\right)\right\rangle=\sqrt{\sum_{k=1}^{n+1} R\left(a_{k}\right)}\left|\psi_{n+1}\left(a_{0}\right)\right\rangle, \\
& A_{-}\left(a_{0}\right)\left|\psi_{n}\left(a_{0}\right)\right\rangle=\sqrt{\sum_{k=0}^{n-1} R\left(a_{k}\right)}\left|\psi_{n-1}\left(a_{0}\right)\right\rangle .
\end{aligned}
$$

As a result, the normalized eigenstates of $H_{0}$ are given by

$$
\left|\psi_{n}\left(a_{0}\right)\right\rangle=\frac{1}{\sqrt{[n]_{0} !}}\left\{A_{+}\left(a_{0}\right)\right\}^{n}\left|\psi_{0}\left(a_{0}\right)\right\rangle
$$

with simplified notations

$$
\begin{aligned}
& {[n]_{k} \equiv R\left(a_{k+1}\right)+R\left(a_{k+2}\right)+\cdots R\left(a_{k+n}\right)} \\
& \widehat{[n]}_{k} \equiv[n]_{k} T \\
& {[n]_{k} ! \equiv \widehat{[n]}_{k}[\widehat{n-1}]_{k} \cdots \widehat{[1]_{k}} \cdot T^{-n}}
\end{aligned}
$$

If $R$ is constants, the spectra are equidistant, and $[n]_{k}$ and $[n]_{k}$ ! reduce to the usual natural number and the factorial, respectively. In general, however, they depend on the parameter $a$, and therefore more complicated situations occur. For example, $[n]_{k}$ does not commute with $T$ in general: $\left[T,[n]_{k}\right]=\left([n]_{k+1}-[n]_{k}\right) T=$ $\left(R\left(a_{k+1+n}\right)-R\left(a_{k+1}\right)\right) T$.

As we obtain the normalized eigenstates, we proceed to construct the coherent states, i.e., the eigenstates of $A_{-}\left(a_{0}\right)$. We assume in the following that the systems under consideration have infinite bound states. Let $\exp _{k}(x)$ be a generalized exponential function defined by the use of the generalized factorial (14)

$$
\exp _{k}(x) \equiv \sum_{n=0}^{\infty} \frac{1}{[n]_{k} !} x^{n}
$$

Then we can define the following states dependent on a complex parameter $z$ as

$$
\begin{aligned}
\left.\mid z, a_{0}\right) & \equiv \exp _{0}\left\{z A_{+}\left(a_{0}\right)\right\}\left|\psi_{0}\left(a_{0}\right)\right\rangle \\
& =\sum_{n=0}^{\infty} \frac{1}{\sqrt{[n]_{0} !}} z^{n}\left|\psi_{n}\left(a_{0}\right)\right\rangle
\end{aligned}
$$

where round ket means an unnormalized state. It may be needless to say that we can define similar coherent states $\left.\mid z, a_{k}\right)$ by replacing $\exp _{0} \rightarrow \exp _{k}$. If we operate 
$A_{-}\left(a_{0}\right)$ to the state $(16)$, we have

$$
\begin{aligned}
\left.A_{-}\left(a_{0}\right) \mid z, a_{0}\right) & =\sum_{n=0}^{\infty} \frac{1}{\sqrt{[n]_{-1} !}} z^{n} A_{-}\left(a_{0}\right)\left|\psi_{n}\left(a_{0}\right)\right\rangle \\
& =\sum_{n=1}^{\infty} \frac{1}{\sqrt{[n]_{-1} T \cdot[n-1]_{-1} ! \cdot T^{-1}}} z^{n} \sqrt{[n]_{-1}}\left|\psi_{n-1}\left(a_{0}\right)\right\rangle \\
& \left.=\sum_{n=1}^{\infty} \frac{1}{\sqrt{T[n-1]_{-1} ! T^{-1}}} z^{n}\left|\psi_{n-1}\left(a_{0}\right)\right\rangle=z \mid z, a_{0}\right) .
\end{aligned}
$$

For a reference' sake, we mention that without using the generalized exponential function we can formally express the coherent state as

$$
\left.\mid z, a_{0}\right)=\left[1-z\left\{\frac{1}{H_{0}} A_{+}\left(a_{0}\right)\right\}\right]^{-1}\left|\psi_{0}\left(a_{0}\right)\right\rangle .
$$

If $R(a)=1$, this state corresponds exactly to the usual boson coherent state. The overlap and norm of this state are given by, from Eq.(16)

$$
\begin{aligned}
& \left(z, a_{0} \mid z^{\prime}, a_{0}\right)=\exp _{0}\left(\bar{z} z^{\prime}\right), \\
& \mathcal{N}_{z}\left(a_{0}\right) \equiv\left(z, a_{0} \mid z, a_{0}\right)=\exp _{0}\left(|z|^{2}\right) .
\end{aligned}
$$

We see that the state (16) is non-orthogonal as the usual coherent state. This property plays a crucial role especially in coherent states path integrals when evaluated in the semiclassical approximation $[10,11]$. Using the norm (19), we obtain the normalized state

$$
\left.\left|z, a_{0}\right\rangle \equiv \frac{1}{\sqrt{\mathcal{N}_{z}\left(a_{0}\right)}} \mid z, a_{0}\right) .
$$

It should be noted that this normalized state has the different eigenvalue from the unnormalized one ;

$$
\sqrt{\frac{\mathcal{N}_{z}\left(a_{0}\right)}{\mathcal{N}_{z}\left(a_{-1}\right)}} z,
$$

since the parameter in the normalization factor is changed by the action of $A_{-}\left(a_{0}\right)$. Up to now, we have examined coherent ket-states. We here refer to the conjugate ones. From $\left.\left.\left.A_{-}\left(a_{1}\right) \mid z, a_{1}\right)=D\left(a_{0}\right) \mid z, a_{0}\right)=z \mid z, a_{1}\right)$, we have the corresponding relation between coherent bra-states $\left(z, a_{0} \mid D^{\dagger}\left(a_{0}\right)=\left(z, a_{1} \mid \bar{z}\right.\right.$, i.e., $\left(z, a_{0} \mid A_{+}\left(a_{0}\right)=\right.$ $\left(z, a_{1} \mid \bar{z} T\right.$. 
Next consider an important property of the coherent state, i.e., the completeness relation. We follow the classification of Ref.[8] and confine our attention to systems with only bound states. Constants $a, b, c, d$ in this reference correspond to $\alpha, \beta, \gamma, \delta$, respectively.

(I) Types (C) and (D). These are the simplest cases among the shape-invariant potentials. Superpotentials $W$ and $R$ in Eq.(1) are given by

$$
\begin{aligned}
& W(x, a)= \begin{cases}(a+\delta) / x+\beta x / 2 & (\mathrm{C}), \\
\beta x+\delta & (\mathrm{D}),\end{cases} \\
& R(a)=\beta,
\end{aligned}
$$

where $\beta$ and $\delta$ are some real constants. In these cases we can set $R(a)=1$ without loss of generality. Then

$$
[n]_{k}=n, \quad[n]_{k} !=n !,
$$

are independent of $k$, namely, they are the usual natural numbers and factorial, respectively. The coherent state becomes, therefore,

$$
\begin{aligned}
& \left.\mid z, a_{0}\right)=\sum_{n=0}^{\infty} \frac{z^{n}}{\sqrt{n !}}\left|\psi_{n}\left(a_{0}\right)\right\rangle, \\
& \mathcal{N}_{z}\left(a_{0}\right)=\exp \left(|z|^{2}\right) .
\end{aligned}
$$

For the measure

$$
d \mu(z)=\frac{1}{\pi} d \operatorname{Re} z d \operatorname{Im} z,
$$

we have the following completeness relation

$$
\begin{aligned}
\int & d \mu(z)\left|z, a_{0}\right\rangle\left\langle z, a_{0}\right| \\
= & \sum_{m, n=0}^{\infty} \frac{1}{\sqrt{m ! n !}}\left|\psi_{m}\left(a_{0}\right)\right\rangle\left\langle\psi_{n}\left(a_{0}\right)\right| \frac{1}{\pi} \int d \operatorname{Re} z d \operatorname{Im} z \exp \left(-|z|^{2}\right) \bar{z}^{m} z^{n} \\
= & \sum_{n=0}^{\infty}\left|\psi_{n}\left(a_{k}\right)\right\rangle\left\langle\psi_{n}\left(a_{k}\right)\right| .
\end{aligned}
$$

(II) Types (A) and (B). Superpotentials and $R$ are given by

$$
\begin{aligned}
& W(x, a)= \begin{cases}\alpha(a+\gamma) \cot \alpha(x+p)+\delta / \sin \alpha(x+p) & (\mathrm{A}) \\
i \alpha(a+\gamma)+\delta \exp (-i \alpha x)\end{cases} \\
& R(a)=-\alpha^{2}\left(a+\gamma+\frac{1}{2}\right)
\end{aligned}
$$


where $\alpha$ is in general a real or pure imaginary constant for type $(\mathrm{A})$, while it is pure imaginary for type $(\mathrm{B})$, and $\gamma, \delta$ and $p$ are real constants. However, as we assume that the hamiltonian has only bound states, we confine our attention to the type (A) with real $\alpha$. Note that in $W$ and $R, \gamma$ always appears as a form $a+\gamma$. Therefore, we can put $\gamma=0$ without loss of generality. Furthermore, $R$ can reduce to $R(a)=-(a+1 / 2)$ by replacing $A_{ \pm} \rightarrow A_{ \pm} / \alpha$. Finally, we can fix the various parameters by choosing $a_{0}$ properly. Among them, for example, if we choose $a_{0}=-1 / 2$, we have a simple expression for the coherent state (16) as follows; first we have

$$
\begin{aligned}
& {[n]_{k}=\frac{1}{2} n(2 k+n+1)} \\
& {[n]_{k} !=\frac{1}{2^{n}} n ! \frac{(2 k+2 n) !}{(2 k+n) !}}
\end{aligned}
$$

It should be noted the factorial depends on $k$, namely, on $a$. For $k=0$, we have $[n]_{0} !=(2 n) ! / 2^{n}$ and

$$
\begin{aligned}
& \left.\mid z, a_{0}\right)=\sum_{n=0}^{\infty} \frac{1}{\sqrt{(2 n) !}} z^{n}\left|\psi_{n}\left(a_{0}\right)\right\rangle \\
& \mathcal{N}_{z}\left(a_{0}\right)=\sum_{n=0}^{\infty} \frac{|z|^{2 n}}{(2 n) !}=\cosh (|z|)
\end{aligned}
$$

where we replace $\sqrt{2} z \rightarrow z$. For the mesure

$$
d \mu_{0}(z)=\frac{1}{2 \pi} \mathcal{N}_{z}\left(a_{0}\right) \frac{\exp (-|z|)}{|z|} d \operatorname{Re} z d \operatorname{Im} z
$$

we have

$$
\begin{aligned}
\int & d \mu_{0}(z)\left|z, a_{0}\right\rangle\left\langle z, a_{0}\right| \\
& =\sum_{m, n=0}^{\infty} \frac{1}{\sqrt{(2 m) !(2 n) !}}\left|\psi_{m}\left(a_{0}\right)\right\rangle\left\langle\psi_{n}\left(a_{0}\right)\right| \frac{1}{2 \pi} \int d \operatorname{Re} z d \operatorname{Im} z \frac{\exp (-|z|)}{|z|} \bar{z}^{m} z^{n} \\
& =\sum_{n=0}^{\infty}\left|\psi_{n}\left(a_{0}\right)\right\rangle\left\langle\psi_{n}\left(a_{0}\right)\right|
\end{aligned}
$$

We can obtain the similar relation for $\left.\mid z, a_{k}\right)$ by transforming the last equation by $T^{k}$. 
(III) Types (E) and (F). Superpotentials and $R$ are given by

$$
\begin{aligned}
& W(x, a)= \begin{cases}\alpha a \cot \alpha(x+p)+q / a & (\mathrm{E}), \\
a / x+q / a & (\mathrm{~F}),\end{cases} \\
& R(a)=-\alpha^{2}\left(a+\frac{1}{2}\right)+\frac{q^{2}}{2}\left\{\frac{1}{(a+1)^{2}}-\frac{1}{a^{2}}\right\}, \quad(\alpha=0 \text { for } \mathrm{F}),
\end{aligned}
$$

where $\alpha$ is in general a real or pure imaginary constant, and $q$ is a real constant. Note that the potentials in type (F) always have continuous states as well as bound states and those in type (E) are able to have only bound states for real $\alpha$. Therefore we restrict ourselves only to this type. In this case we may also construct the coherent state based on Eq.(16) in the same way as the above. In an alternative way, to make use of the discussion in (II), we can consider another coherent state by using, instead of $A_{ \pm}$, newly defined operators,

$$
\widetilde{A}_{+}(a) \equiv A_{+}(a)-\frac{q}{\sqrt{2} a} T, \quad \widetilde{A}_{-}(a) \equiv A_{-}(a)-T^{-1} \frac{q}{\sqrt{2} a},
$$

which satisfy $\left[\widetilde{A}_{-}, \widetilde{A}_{+}\right]=\widetilde{R}$ with $\widetilde{R}(a)=-\alpha^{2}(a+1 / 2)$. Using these operators, we can define a coherent state in the same properties as the category (II).

In summary, by introducing an operator $T$ denoting a reparametrization of $a$, we have represented the shape-invariance condition as a form of a commutation relation, and by using it we have constructed a coherent state associated with the shape-invariant potentials. We expect this state should play a similar role as the usual and generalized coherent states have been playing in various field in modern physics.

Finally, we note that in types (C) and (D), the commutation relation (7) is closed and is considered a non-unitary realization of the Heisenberg-Weyl algebra. In types (A) and (B), we can also rewrite Eq.(7) in a closed form as follows:

$$
\left[K_{+}(a), K_{-}(a)\right]=-2 K_{0}(a), \quad\left[K_{0}(a), K_{ \pm}(a)\right]= \pm K_{ \pm}(a)
$$

where

$$
K_{+}(a)=\sqrt{\frac{2}{\alpha}} A_{+}(a), \quad K_{-}(a)=\sqrt{\frac{2}{\alpha}} A_{-}(a), \quad K_{0}(a)=\frac{R(a)}{\alpha} .
$$

Then we can consider that shape-invariance condition is a non-unitary realization of the $s u(1,1)$ algebra for the cases (A) and (B). The hamiltonian is given simply 
by $H_{0} \propto K_{+} K_{-}$. We see that the shape-invariant potentials have a quite simple algebraic structure. However, one should note that $K_{ \pm}$do not commute with a

function $f(a):\left[K_{ \pm}, f(a)\right]=\left(f\left(a_{1}\right)-f(a)\right) K_{ \pm}$. Therefore, some unusual features may occur in the representation theory. For example, we can construct a Casimir operator which commute with all generators of Eq.(35). However, its eigenvalues are not commutative with these generators since they depend on $a$.

It may be interesting to consider a possibility of new shape-invariant potentials based on some other algebras.

\section{REFERENCES}

1. For reviews of coherent states, see, e.g., J. R. Klauder "A Coherent-State Primer" in Coherent States. Applicatons in Physics and Mathmatical Physics, eds. J. R. Klauder and B. S. Skagerstam (World Scientific, Singerpore, 1985); A. Perelomov, Generalized Coherent States and Their Applications (SpringerVerlag, Berlin, 1986).

2. J. R. Klauder, Phys. Rev. D19 (1979) 2349.

3. H. Kuratsuji and T. Suzuki, J. Math Phys. 21 (1980) 472.

4. R. G. Littlejohn, Phys. Rep. 138 (1986) 193, and references therein.

5. T. Fukui, Prog. Theor. Phys. 87 (1992) 927.

6. L. E. Gendenstein, JETP Lett. 38 (1983) 356; L. E. Gendenstein and I. V. Krive, Sov. Phys. Usp. 28 (1985) 645.

7. E. Witten, Nucl. Phys. B185 (1981) 513.

8. L. Infeld and T. E. Hull, Rev. Mod. Phys. 23 (1951) 21.

9. V. Spiridonov, Phys. Rev. Lett. 69 (1992) 398.

10. T. Suzuki, Nucl. Phys. 398 (1983) 398.

11. T. Fukui, RCNP preprint 044.

The level scheme 